\title{
The Dynamic Equation and Simulation of a Linkage Mechanism Fabricated from Three-Dimensional Braided Composite Materials*
}

\author{
Gan-wei CAI**, Ru-gui WANG** and Zhao-jun LI $^{* * *}$
}

\begin{abstract}
The work in this paper is concerned with a four-bar linkage mechanism with links fabricated from three-dimensional braided composite materials. The dynamic equation of the mechanism is established by finite element method (FEM), it includes composite materials parameters and structural parameters of the mechanism. The mass matrix of the beam element is obtained in light of the mass distribution characteristics of the composite materials. According to the 3-dimensional microstructure characters of the three-dimensional braided composite materials, the anisotropic beam element is considered to be made up of four parts, and then the stiffness matrix of the beam element is derived from the constitutive equations of each part and the relation between the strain distribution of each part and the node displacement of the beam element. Based on the damping element model and the expression of dissipation energy of the 3-dimentional braided composite materials, each modal damping value of the mechanism is calculated and the damping matrix of the mechanism is established. The dynamic responses and natural frequency of the mechanism are obtained by simulation, respectively. The work presented in the paper provides theoretical basis to a certain extent for the further research on nonlinear vibration characteristics and optimum design of this kind of mechanism.
\end{abstract}

Key Words: Elastic Mechanism, Finite Element Method, Composite Materials, Dynamic Analysis

\section{Introduction}

The development of mechanology has a close connection with the invention and application of new materials, and the research on the application of new materials is very important in the domain of modern mechanology. It is one of the valid strategies improving the elastodynamic properties of mechanical systems to exploit the advanced composite materials of which the high strength-to-weight ratios and damping properties are superior to that of the conventional metals.

In the 80's in last century, people began to study the problem about the application of composite laminates to

* Received 25th January, 2006 (No. 05-5146)

** College of Mechanical Engineering, Guangxi University, Nanning 530004, Guangxi, P.R. China.

E-mail: cganwei@gxu.edu.cn; caiganwei@163.com

*** School of Mechanical Science \& Engineering, Huazhong University of Science \& Technology, Wuhan 430074, Hubei, P.R. China linkage mechanisms. And then, the mechanisms with links fabricated from composite laminates have been applied gradually into many fields such as high-speed production lines and the industrial robots, and it is shown in engineering practice that this kind of mechanism has some advantages such as lightweight and short residual vibration's time ${ }^{(1)-(5)}$. But the composite laminates have a grievous disfigurement that the strength between layers is very low and their delaminations occur frequently. The three-dimensional braided fiber-reinforced composite material is a new type of composite material. In comparing with two-dimensional composite laminates, a key property of the innovative three-dimensional braided composite is its ability to reinforce composites in the thickness. Unlike convention laminates, it includes multi-directional fiber bundles which interconnect layers. This fiber construction reduces the usual tendency toward delamination ${ }^{(6)-(13)}$.

As can be seen from above, there is high potential of three-dimensional braided composite applications in highspeed linkage mechanisms. In this paper, a four-bar link- 
age mechanism fabricated from three-dimensional braided composite materials is studied, and its dynamic equation is established by the FEM. The mass matrix of the beam element is obtained in light of the mass distribution characteristics of the composite materials. According to the 3-dimensional microstructure characters of the threedimensional braided composite materials, the anisotropic beam element is considered to be made up of four parts, and then the stiffness matrix of the beam element is derived from the constitutive equations of each part and the relation between the strain distribution of each part and the node displacement of the beam element. Based on the damping element model and the expression of dissipation energy of the 3-dimentional braided composite materials, each modal damping value of the mechanism is calculated and the damping matrix of the mechanism is established. The dynamic responses and natural frequency of the mechanism are obtained by simulation, respectively. The work presented in the paper provides theoretical basis to a certain extent for the further research on nonlinear vibration characteristics and optimum design of this kind of mechanism.

In order to simplify the discussion and reveal the leading problems, the instantaneous structure hypothesis $^{(14),(15)}$ of kineto-elastodynamics (KED) is adopted in analysis. At the same time, the damping of joints is neglected, for the KED is mainly used to study the elastic deformation of the links of mechanism

\section{Mass Matrix and Stiffness Matrix of the Mechan- ism}

The basic fiber structure of the 3-dimensional braided composite materials is of 3-dimention and 4-direction texture, as shown in Fig. 1, and the geometrical unit-cell can be adopted in analysis, as shown in Fig. 2. Four bundles of braided yarns are in diagonal direction of the unit-cell, and intersect at the center point $\mathrm{O}$. The angle $\alpha$ between each braided yarn direction and $x$-axis direction is called the braided angle, and $\beta$ is the angle between the direction of projection of each braided yarn on the $Y-Z$ plane and $y$-axis direction. The $\alpha$ and $\beta$ are shown in Fig. 2 are belong to the first direction braided yarn. According to the laminated plate analogy theory, the 3-dimesional braided composite materials can be regarded as the superposition of four unidirectional fiber composite materials, and the stiffness and damping of the materials are respectively the superposition of stiffness and damping of the four parts according to their contributing volume fractions ${ }^{(6)-(13)}$.

Consider the bending moment, shearing force and axial force, the displacement field of any point of composite link can be expressed as follows ${ }^{(11),(12),(16)}$ :

$$
V(x, z)=V_{0}(x)+z \varphi(x), W(x, z)=W_{0}(x)
$$

where $V_{0}(x)$ and $W_{0}(x)$ are the longitudinal and transverse displacements of the neutral layer of link, respectively.

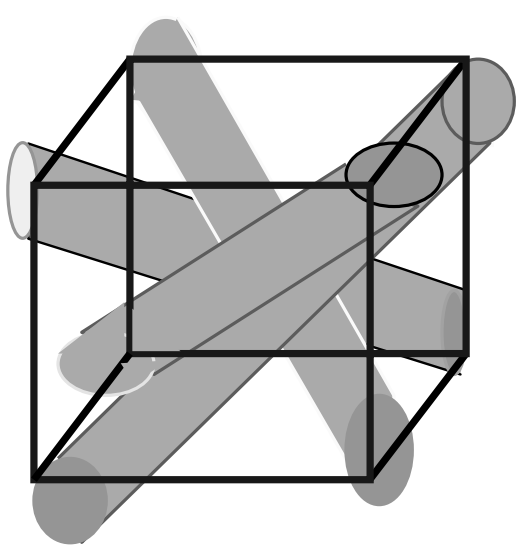

Fig. 1 3-dimention and 4-direction structure of the 3-dimentional braided composite material

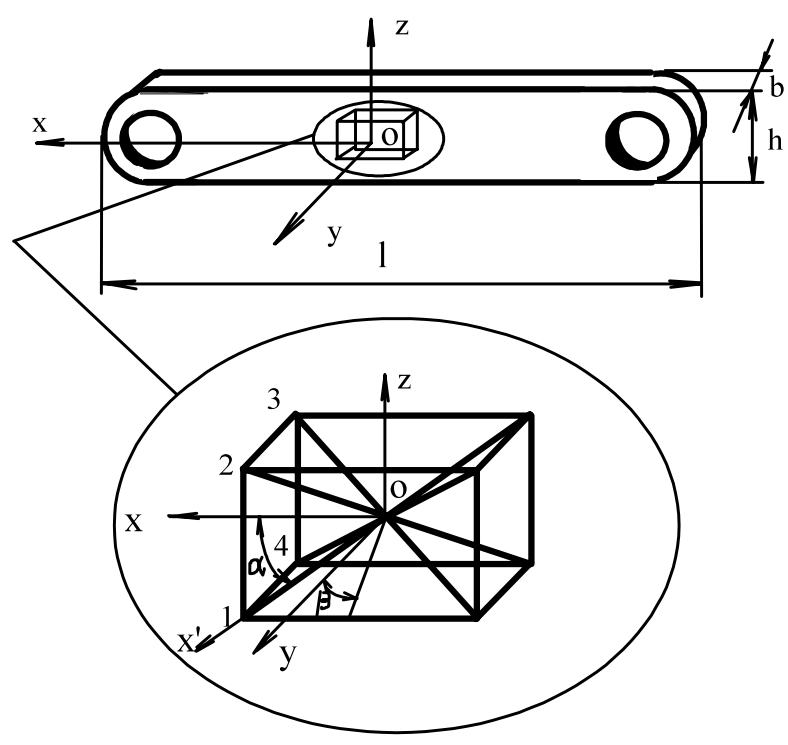

Fig. 2 Geometrical unit cell

$\varphi(x)$ is the angle displacement of cross-section of link. From elasticity theory, the strain-displacement relationship of composite links can be expressed as ${ }^{(11),(12),(16),(17) \text { : }}$

$$
\varepsilon_{x}=\frac{\partial V_{0}(x)}{\partial x}+z \frac{\partial \varphi(x)}{\partial x}, \gamma_{x z}=\frac{\partial W_{0}(x)}{\partial x}+\varphi(x)
$$

\subsection{Element shape function}

In general, the links of the linkage mechanism are slim bars, so they are adequate to be simulated using the beam element. As shown in Fig. 3, the 3-dimensional braided composite beam element has two nodes (A and $\mathrm{B})$, and the origin of the local coordinate system for the beam element is assumed to be at the point $\mathrm{A}$. The displacements of any point and each cross-sectional rotation angle within the element can be expressed as in terms of the shape function and nodal displacements:

$$
\begin{cases}V(\bar{x}, t)=\sum_{i} \phi_{i}(\bar{x}) u_{i}(t) & (i=1,5) \\ W(\bar{x}, t)=\sum_{i} \phi_{i}(\bar{x}) u_{i}(t) & (i=2,3,4,6,7,8) \\ \varphi(\bar{x}, t)=\sum_{i} \phi_{i}(\bar{x}) u_{i}(t) & (i=9,10)\end{cases}
$$




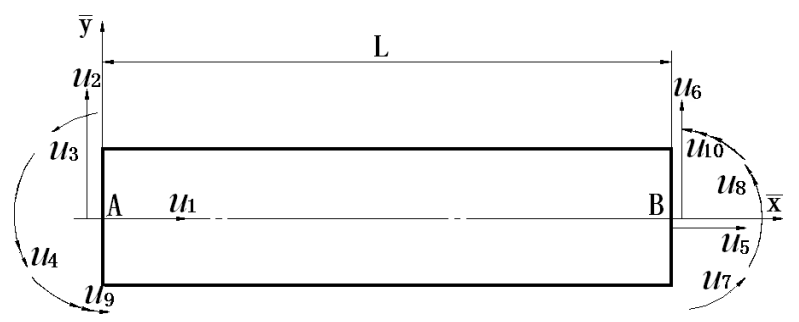

Fig. 3 The beam element model of composite material

where $\bar{x}$ is the coordinate of the beam element in the local coordinate system, $u_{1}$ and $u_{5}$ are the longitudinal displacements of the nodes, $u_{2}$ and $u_{6}$ are the transverse displacements of the nodes, $u_{3}$ and $u_{7}$ are the elastic rotation angles of axes at the nodes, $u_{4}$ and $u_{8}$ are the curvatures of axes at the nodes, $u_{9}$ and $u_{10}$ are the cross-sectional rotation angle at the nodes, and the generalized coordinates vector of the beam element is:

$$
\boldsymbol{u}=\left[\begin{array}{llllllllll}
u_{1} & u_{2} & u_{3} & u_{4} & u_{5} & u_{6} & u_{7} & u_{8} & u_{9} & u_{10}
\end{array}\right]^{T}
$$

The shape functions $\phi_{i}(\bar{x})$ in Eq. (3) are given by

$$
\left\{\begin{array}{l}
\phi_{1}(\bar{x})=1-e \\
\phi_{2}(\bar{x})=1-10 e^{3}+15 e^{4}-6 e^{5} \\
\phi_{3}(\bar{x})=L \times\left(e-6 e^{3}+8 e^{4}-3 e^{5}\right) \\
\phi_{4}(\bar{x})=L^{2} \times\left(e^{2}-3 e^{3}+3 e^{4}-5 e^{5}\right) / 2 \\
\phi_{5}(\bar{x})=e \\
\phi_{6}(\bar{x})=10 e^{3}-15 e^{4}+6 e^{5} \\
\phi_{7}(\bar{x})=L \times\left(-4 e^{3}+7 e^{4}-3 e^{5}\right) \\
\phi_{8}(\bar{x})=L^{2} \times\left(e^{3}-2 e^{4}+e^{5}\right) / 2 \\
\phi_{9}(\bar{x})=1-e \\
\phi_{10}(\bar{x})=e
\end{array}\right.
$$

where $L$ is the length of the beam element, $0 \leq \bar{x} \leq L$, and $e=\frac{\bar{x}}{L}$.

\subsection{Kinetic energy of element}

Considering the moment of inertia, the total kinetic energy $T$ of the element equals the sum of the translational kinetic energy $T_{1}$ and the rotational energy $T_{2}$.

$$
T=T_{1}+T_{2}
$$

where

$$
\left\{\begin{array}{l}
T_{1}=\frac{1}{2} \int_{0}^{L} m\left(\left[\dot{V}_{a}(\bar{x}, t)\right]^{2}+\left[\dot{W}_{a}(\bar{x}, t)\right]^{2}\right) d \bar{x} \\
T_{2}=\frac{1}{2} \int_{0}^{L} I \dot{\varphi}_{a}^{2}(\bar{x}, t) d \bar{x}
\end{array}\right.
$$

where, $\dot{V}_{a}(\bar{x}, t)$ and $\dot{W}_{a}(\bar{x}, t)$ are respectively the instantaneous absolute velocities of the central point of any cross section of element in the longitudinal and transverse directions, $\dot{\varphi}_{a}$ is the absolute angular velocity. $m$ and $I$ are the cross sectional mass and moment of inertia, respectively, and

$$
(m, I)=\iint_{S}\left(1, z^{2}\right) \rho d S
$$

Where $\rho$ is the average mass density of materials, and $\rho=$ $\rho_{f} v_{f}+\rho_{m} v_{m}, \rho_{f}$ and $\rho_{m}$ are respectively the densities of fiber and matrix, $v_{f}$ and $v_{m}$ are respectively the volume percent of fiber and matrix, respectively, $S$ is the area of the cross-section.

Substituting Eqs. (3) and (5) into Eq. (7), the translational kinetic energy $T_{1}$ of the element is:

$$
T_{1}=\frac{1}{2} \dot{\boldsymbol{u}}_{a}^{T} \overline{\boldsymbol{M}}_{1} \dot{\boldsymbol{u}}_{a}
$$

where $\dot{\boldsymbol{u}}_{a}$ is the absolute velocity vector of nodes, $\overline{\boldsymbol{M}}_{1}$ is the inertia matrix related to the length $L$ of the element.

$$
\left\{\begin{array}{c}
\left(\bar{m}_{1}\right)_{i j}=\rho S \int_{0}^{L} \phi_{i}(\bar{x}) \phi_{j}(\bar{x}) d \bar{x} \\
(i, j=1,2,3,4,5,6,7,8) \\
\left(\bar{m}_{1}\right)_{i j}=0 \\
(i, j=9,10)
\end{array}\right.
$$

The rotational energy $T_{2}$ of the element is:

$$
T_{2}=\frac{I}{2} \int_{0}^{L}[\dot{\theta}(t)+\dot{\varphi}(\bar{x}, t)]^{2} d \bar{x}=\frac{1}{2} I \dot{\theta} L+\frac{1}{2} \dot{\boldsymbol{u}}^{T} \overline{\boldsymbol{M}}_{2} \dot{\boldsymbol{u}}+\dot{\boldsymbol{u}} \boldsymbol{F}
$$

where $\theta$ is the angular displacement of the rigid beam relative to the fixed coordinate system, $\varphi$ is the elastic angular displacement of the cross-section relative to the moving coordinate system, $\dot{\boldsymbol{u}} \boldsymbol{F}$ is the coupling of rigid motion and elastic motion, it is neglected in this paper. The inertia matrix $\overline{\boldsymbol{M}}_{2}$ is

$$
\left\{\begin{array}{c}
\left(\bar{m}_{2}\right)_{i j}=\frac{I}{2} \int_{0}^{L} \phi_{i}(\bar{x}) \phi_{j}(\bar{x}) d \bar{x} \\
(i, j=9,10) \\
\left(\bar{m}_{2}\right)_{i j}=0 \\
(i, j=1,2,3,4,5,6,7,8)
\end{array}\right.
$$

Therefore, inertia matrix of the beam element is:

$$
\overline{\boldsymbol{M}}=\sum_{j=1}^{2} \overline{\boldsymbol{M}}_{j}
$$

\subsection{Potential energy of element}

As described above, the 3-dimensional braided composite materials can be regarded as the superposition of four unidirectional fiber composite materials. The strain energy of the $k$-th part in the beam element can be expressed as:

$$
W_{k}=\frac{1}{2} \int_{\Omega} \boldsymbol{\varepsilon}_{k}^{T} \sigma_{k} d \Omega
$$

where $\Omega$ is a quarter of the element volume. $\boldsymbol{\varepsilon}_{k}$ and $\boldsymbol{\sigma}_{k}$ are respectively the strain and stress vector of the $k$-th part in its principal material orientation, respectively, and they can be expressed as ${ }^{(17)}$ :

$$
\left\{\begin{array}{l}
\boldsymbol{\varepsilon}_{k}^{T}=\left[\varepsilon_{x^{\prime}}, \varepsilon_{y^{\prime}}, \varepsilon_{z^{\prime}}, \gamma_{y^{\prime} z^{\prime}}, \gamma_{z^{\prime} x^{\prime}}, \gamma_{x^{\prime} y^{\prime}}\right] \\
\sigma_{k}=\left[\sigma_{x^{\prime}}, \sigma_{y^{\prime}}, \sigma_{z^{\prime}}, \tau_{y^{\prime} z^{\prime}}, \tau_{z^{\prime} x^{\prime}}, \tau_{x^{\prime} y^{\prime}}\right]^{T}
\end{array}\right.
$$

Each part can be considered as transversely isotropy, so the constitutive relation of the $k$-th part in its principal material orientation can be expressed as ${ }^{(13),(17)}$

$$
\sigma_{k}=\boldsymbol{H} \boldsymbol{\varepsilon}_{k}
$$




$$
\boldsymbol{H}=\left(\begin{array}{ccccccc}
H_{11} & H_{12} & H_{13} & & & \\
H_{12} & H_{22} & H_{23} & & 0 & \\
H_{13} & H_{23} & H_{33} & & & \\
& & & H_{44} & & \\
& 0 & & & H_{55} & \\
& & & & & H_{66}
\end{array}\right)
$$

where $^{(13),(17)}$

$$
\begin{aligned}
& H_{11}=\frac{\left(1-\mu_{23} \mu_{23}\right) E_{11}}{K} ; H_{12}=H_{13}=\frac{\left(1+\mu_{23}\right) \mu_{21} E_{11}}{K} ; \\
& H_{22}=H_{33}=\frac{\left(1-\mu_{21} \mu_{12}\right) E_{22}}{K} ; \\
& H_{23}=\frac{\left(\mu_{23}+\mu_{12} \mu_{21}\right) E_{22}}{K} ; \\
& H_{44}=H_{23} ; \quad H_{55}=H_{13} ; H_{66}=H_{12} ; \\
& K=1-2 \mu_{12} \mu_{21}\left(1+\mu_{23}\right)-\mu_{23} \mu_{23} .
\end{aligned}
$$

According to micromechanics of composite materials, the elastic constants $E_{i j}, G_{i j}$ and $\mu_{i j}$ can be calculated from elastic constants of fiber and matrix, volume ratio of fiber and the microstructure parameters of composite materi$\operatorname{als}^{(13),(17)}$.

The strains of the $k$-th part in the beam coordinate system XYZ can be obtained from the transition relation of strain in elastic mechanics as ${ }^{(13),(17)}$

$$
\boldsymbol{\varepsilon}_{k}=\boldsymbol{L} \boldsymbol{\varepsilon}
$$

where the transition matrix $\boldsymbol{L}$ of strain is determined by the direction cosine between the beam coordinate system $\mathrm{XYZ}$ and the coordinate system $\mathrm{X}^{\prime} \mathrm{Y}^{\prime} \mathrm{Z}^{\prime}$ of $k$-th part in its principal material orientation.

Substituting Eqs. (3)-(5) into Eq. (2), the relations between the strains and nodal displacements can be written as

$$
\varepsilon=S u
$$

where

$$
\left\{\begin{aligned}
S= & {\left[S_{1}, S_{2}, S_{3}, S_{4}, S_{5}, S_{6}, S_{7}, S_{8}, S_{9}, S_{10}\right]^{T} } \\
S_{1} & =\left[-\frac{1}{L}, 0,0,0, \frac{1}{L}, 0,0,0,-\frac{z}{L}, \frac{z}{L}\right] \\
S_{5} & =\left[0,-\frac{30 x^{2}}{L^{3}}+\frac{60 x^{3}}{L^{4}}-\frac{30 x^{4}}{L^{5}}, 1-\frac{18 x^{2}}{L^{2}}\right. \\
& +\frac{32 x^{3}}{L^{3}}-\frac{15 x^{4}}{L^{4}}, x-\frac{9 x}{2 L}+\frac{12 x^{3}}{2 L^{2}}-\frac{25 x^{4}}{2 L^{3}}, 0, \\
& \frac{30 x^{2}}{L^{3}}-\frac{60 x^{3}}{L^{4}}+\frac{30 x^{4}}{L^{5}},-\frac{12 x^{2}}{L^{2}}+\frac{28 x^{3}}{L^{3}} \\
& \left.-\frac{15 x^{4}}{L^{4}}, \frac{3 x^{2}}{L}-\frac{8 x^{3}}{L^{2}}+\frac{5 x^{4}}{L^{3}}, 1-\frac{x}{L}, \frac{x}{L}\right] \\
\boldsymbol{S}_{2}= & \boldsymbol{S}_{3}=\boldsymbol{S}_{4}=\boldsymbol{S}_{6}=\boldsymbol{S}_{7}=\boldsymbol{S}_{8}=\boldsymbol{S}_{9}=\boldsymbol{S}_{10}=\mathbf{0} \\
\boldsymbol{u} & =\left[u_{1}, u_{2}, u_{3}, u_{4}, u_{5}, u_{6}, u_{7}, u_{8}, u_{9}, u_{10}\right]^{T}
\end{aligned}\right.
$$

Substituting Eqs. (15)-(20) into Eq. (14), the strain energy of the $j$-th part is:

$$
W_{j}=\frac{1}{2} \int_{\Omega} \boldsymbol{u}^{T} \boldsymbol{S}^{T} \boldsymbol{L}^{T} \boldsymbol{H} \boldsymbol{L S} \boldsymbol{u} d \Omega=\frac{1}{2} \boldsymbol{u}^{T} \overline{\boldsymbol{K}}_{j} \boldsymbol{u}
$$

Then the total potential energy of element can be expressed as

$$
W=\sum_{j=1}^{4} W_{j}=\frac{1}{2} \boldsymbol{u}^{T} \overline{\boldsymbol{K}} \boldsymbol{u}
$$

where the stiffness matrix $\overline{\boldsymbol{K}}$ of element is:

$$
\overline{\boldsymbol{K}}=\sum_{j=1}^{4} \overline{\boldsymbol{K}}_{j}
$$

\subsection{Mass matrix and stiffness matrix of the linkage mechanism}

Assuming that $\boldsymbol{R}_{i}$ is the transformation matrix between the $i$-th element coordinates and the global coordinates, the equivalent mass matrix and equivalent stiffness matrix of the $i$-th element in the global coordinate system can be written as ${ }^{(14)-(16)}$

$$
\overline{\boldsymbol{M}}_{i}=\boldsymbol{R}_{i}^{T} \overline{\boldsymbol{M}} \boldsymbol{R}_{i}, \quad \overline{\boldsymbol{K}}_{i}=\boldsymbol{R}_{i}^{T} \overline{\boldsymbol{K}} \boldsymbol{R}_{i}
$$

Assuming that $\boldsymbol{B}_{i}$ is the coordinate compatible matrix of the $i$-th element between the local number and the global number, the mass and stiffness of the $i$-th element in the global coordinates are, respectively ${ }^{(14)-(16)}$ :

$$
\boldsymbol{M}_{i}=\boldsymbol{B}_{i}{ }^{T} \overline{\boldsymbol{M}}_{i} \boldsymbol{B}_{i}, \quad \boldsymbol{K}_{i}=\boldsymbol{B}_{i}{ }^{T} \overline{\boldsymbol{K}}_{i} \boldsymbol{B}_{i}
$$

Therefore, the mass matrix and stiffness matrix of the elastic linkage mechanism can be expressed as:

$$
M=\sum_{i=1}^{N_{e}} M_{i}, K=\sum_{i=1}^{N_{e}} K_{i}
$$

where $N_{e}$ is the element number.

\subsection{Natural frequency and vibration mode of the mechanism}

Under the free vibration circumstances, the Lagrange equation is established by Eq. (26), and the standard eigenvalue equation can be written as

$$
\left(\boldsymbol{K}-\omega^{2} \boldsymbol{M}\right) \boldsymbol{U}=\mathbf{0}
$$

Each order modal natural frequency $\omega_{r}$ and the vibration mode $\boldsymbol{U}$ can be obtained by Eq. (27). $\boldsymbol{U}$ is the vector of elastic displacements of the nodes in the global coordinates. Using $\boldsymbol{U}$, the strain energy $W_{m}$ of mechanism can be obtained as follows:

$$
W_{m}=\frac{1}{2} \boldsymbol{U}^{T} \boldsymbol{K} \boldsymbol{U}
$$

\section{Damping Matrix of the Mechanism}

$$
\psi=\frac{\Delta W}{W_{m}}
$$

where $\Delta W$ is the dissipated energy during a cycle of vibration and $W_{m}$ is the maximum strain energy in the cycle. 


\subsection{Element damping model}

The 3-dimensional braided composite materials can be regarded as the superposition of four part of unidirectional fiber composite materials. The unit dissipation energy of each part should divide into six components, namely

$$
\Delta W_{k}=\Delta W_{1}+\Delta W_{2}+\Delta W_{3}+\Delta W_{4}+\Delta W_{5}+\Delta W_{6}
$$

According to the definition of SDC, every term in Eq. (30) can be expressed as

$$
\Delta W_{i}=\frac{1}{2} \psi_{i} \varepsilon_{i} \sigma_{i}
$$

where, $\psi_{1}, \psi_{2}=\psi_{3}$ are respectively the SDC of the unidirectional composite laminates in the direction $0^{\circ}$ and $90^{\circ}$ when it is bending, $\psi_{4}$ is the SDC of its transverse shearing, $\psi_{5}=\psi_{6}$ is the SDC of its vertical shearing, these $\psi_{i}$ are determined by experiment or selected from the concerned data.

\subsection{Expression of dissipation energy}

Considering Eq. (31), the dissipation energy of the $k$ th part in the beam element can be expressed as

$$
\Delta W_{k}=\frac{1}{2} \int_{\Omega} \boldsymbol{\varepsilon}_{k}^{T} \psi \sigma_{k} d \Omega
$$

where the damping matrix is

$$
\psi=\operatorname{diag}\left[\psi_{1}, \psi_{2}, \psi_{3}, \psi_{4}, \psi_{5}, \psi_{6}\right]
$$

Assuming that $\boldsymbol{Q}_{k}=\boldsymbol{S}^{T} \boldsymbol{L}_{k}^{T} \boldsymbol{\psi} \boldsymbol{H}_{k} \boldsymbol{L}_{k} \boldsymbol{S}$, then Eq. (32) can be written as

$$
\Delta W_{k}=\frac{1}{2} \int_{\Omega} \boldsymbol{u}^{T} \boldsymbol{Q}_{k} \boldsymbol{u} d \Omega=\frac{1}{2} \boldsymbol{u}^{T} \boldsymbol{K}_{d k} \boldsymbol{u}
$$

where

$$
\boldsymbol{K}_{d k}=\int_{\Omega} \boldsymbol{Q}_{k} d \Omega
$$

Therefore, the total dissipation energy of the beam element can be expressed as

$$
\Delta \bar{W}=\sum_{k=1}^{4} \Delta W_{k}=\frac{1}{2} \boldsymbol{u}^{T} \boldsymbol{K}_{d i} \boldsymbol{u}
$$

The damped stiffness matrix of mechanism can be expressed as

$$
\boldsymbol{K}_{d}=\sum \boldsymbol{B}_{i}^{T} \boldsymbol{R}_{i}^{T} \boldsymbol{K}_{d i} \boldsymbol{R}_{i} \boldsymbol{B}_{i}
$$

Each order modal dissipated energy can be obtained by the characteristic vector $\boldsymbol{U}$ derived from Eq. (27) as

$$
\Delta W=\frac{1}{2} \boldsymbol{U}^{T} \boldsymbol{K}_{d} \boldsymbol{U}
$$

When damping is small, the damping ratio $\xi_{r}$ of each order mode of a linear system can be expressed as $^{(16),(18),(19)}$

$$
\xi_{r}=\frac{\psi_{r}}{4 \pi}
$$

Marshaling all modal vectors, the modal matrix of the mechanism can be written as

$$
\boldsymbol{U}=\left[\begin{array}{llll}
\boldsymbol{U}^{(1)} & \boldsymbol{U}^{(2)} & \cdots & \boldsymbol{U}^{(n)}
\end{array}\right]
$$

The normalized modal matrix satisfies the orthogonal conditions as

$$
\begin{aligned}
& \boldsymbol{U}^{T} \boldsymbol{M U}=\boldsymbol{I} \\
& \boldsymbol{U}^{T} \boldsymbol{K} \boldsymbol{U}=\left[\begin{array}{lll} 
& & \\
& \omega_{r}^{2} & \\
& & \backslash
\end{array}\right]
\end{aligned}
$$

When damping is small $\left(\xi_{r} \leq 0.2\right)$, the linear system

$$
\boldsymbol{U}^{T} \boldsymbol{C} \boldsymbol{U}=\left[\begin{array}{llll} 
& & & \\
& 2 \xi_{r} \omega_{r} & \\
& & \backslash
\end{array}\right]
$$

Next we Premultiply Eq. (42) by $\left(\boldsymbol{U}^{T}\right)^{-1}$ and postmultiply Eq. (42) by $\boldsymbol{U}^{-1}$, the damping matrix of mechanism can be expressed as

$$
\boldsymbol{C}=\left(\boldsymbol{U}^{T}\right)^{-1}\left[\begin{array}{lll}
\backslash & & \\
& 2 \xi_{r} \omega_{r} & \\
& & \backslash
\end{array}\right] \boldsymbol{U}^{-1}
$$

considering Eq. (40), we obtain

$$
\boldsymbol{C}=\boldsymbol{M U}\left[\begin{array}{llll}
\backslash & & \\
& 2 \xi_{r} \omega_{r} & \\
& & \backslash
\end{array}\right] \boldsymbol{U}^{T} \boldsymbol{M}
$$

\section{Dynamic Equation of the Mechanism}

Assuming that $\boldsymbol{P}$ and $\boldsymbol{Q}$ are the external exciting force vector and inertial force vector of rigid body, respectively, let $\boldsymbol{U}$ represent the elastic displacement vector in the global coordinates. Incorporating the damping matrix term, the dynamic equation of mechanism can be expressed as:

$$
M \ddot{U}+C \dot{U}+K \boldsymbol{U}=P+Q
$$

The Eq. (45) can be solved by the mode superposition method.

\section{Illustrative Example}

The four-bar linkage with links fabricated form 3dimensional braided composites is shown in Fig. 4. The 3-dimensional braided composite materials consist of carbon fiber and epoxy matrix, the material performance parameters are as follows:

$G_{m}=1.26 \mathrm{GPa}, \mu_{\mathrm{f}}=0.25, \mu_{\mathrm{m}}=0.35, \psi_{1}=0.0045$, $\psi_{2}=\psi_{3}=0.042, \psi_{4}=0.01, \psi_{5}=\psi_{6}=0.0705, \rho_{\mathrm{f}}=$ $1.76 \mathrm{~g} / \mathrm{cm}^{3}, \rho_{\mathrm{m}}=1.36 \mathrm{~g} / \mathrm{cm}^{3}$, the total volume ratio of fiber is 0.6 , the braiding structural parameters are $\alpha=30^{\circ}$ and $\beta=45^{\circ}$

The geometrical parameters of the four-bar linkage mechanism: $L, b$ and $h$ represent the length, width and thickness of the links, respectively; the cross sectional parameters of every link: $b=20 \mathrm{~mm}, h=25 \mathrm{~mm}$, crank $L_{1}=260 \mathrm{~mm}$, coupler $L_{2}=425 \mathrm{~mm}$, rocker $L_{3}=460 \mathrm{~mm}$, frame $L_{4}=620 \mathrm{~mm}$. 


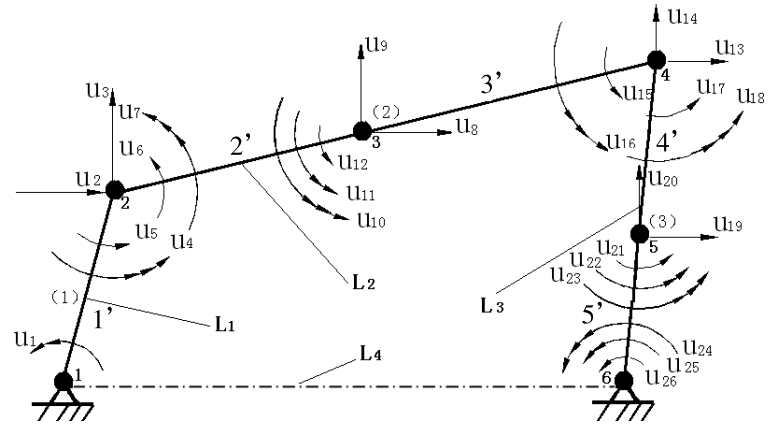

Link $\mathrm{L}_{1}$-Crank; Link $\mathrm{L}_{2}$-Coupler; Link $\mathrm{L}_{3}$-Rocker， Link $\mathrm{L}_{4}$-Frame

Fig. 4 Diagram of linkage mechanism

Table 1 The first four orders natural frequency $\omega_{r}$ and the modal damping $\psi_{r}$ of the mechanism while the angle of crank $\theta_{1}=80^{\circ}$

\begin{tabular}{c|c|c|c|c}
\hline$\omega_{r}(\mathrm{~Hz})$ & 90.5 & 225 & 453 & 1294.1 \\
\hline$\psi_{r}$ & 0.0761 & 0.0518 & 0.0137 & 0.0428 \\
\hline
\end{tabular}

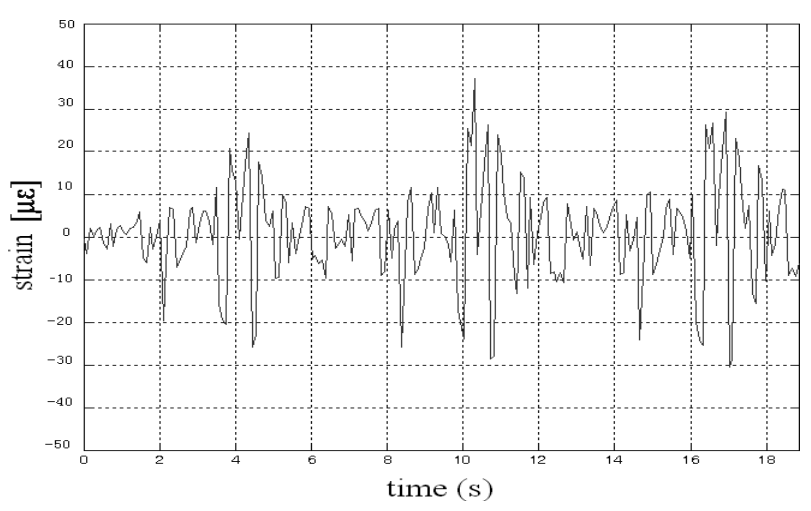

Fig. 5 Simulation time-domain dynamic response characteristics curve of the midpoint of the coupler while $\omega_{1}=$ $300 \mathrm{rpm}$

We regard the crank as one beam element, and regard the rocker and the coupler link as two elements respectively. 1,2,3,4,5 and 6 express the serial number of nodes, 1', 2', 3', 4' and 5' express the serial number of elements, and (1), (2) and (3) express the serial number of bars.

The single arrows represent the elastic displacements and the axis inclination, the dual arrows express the curvature of axis, and the triple arrows stand for the crosssectional rotation angle, as shown in Fig. 4.

The natural frequency $\omega_{r}$ and the modal damping $\psi_{r}$ of the mechanism can be obtained by using the dynamic equation of the mechanism presented in this paper, Table 1 shows the from first to fourth order $\omega_{r}$ and $\psi_{r}$ while the angle of crank $\theta_{1}=80^{\circ}$.

Don't consider the external exciting force, namely $\boldsymbol{P}=0$, the simulation calculation of dynamic response of the mechanism is obtained by the real mode superposition

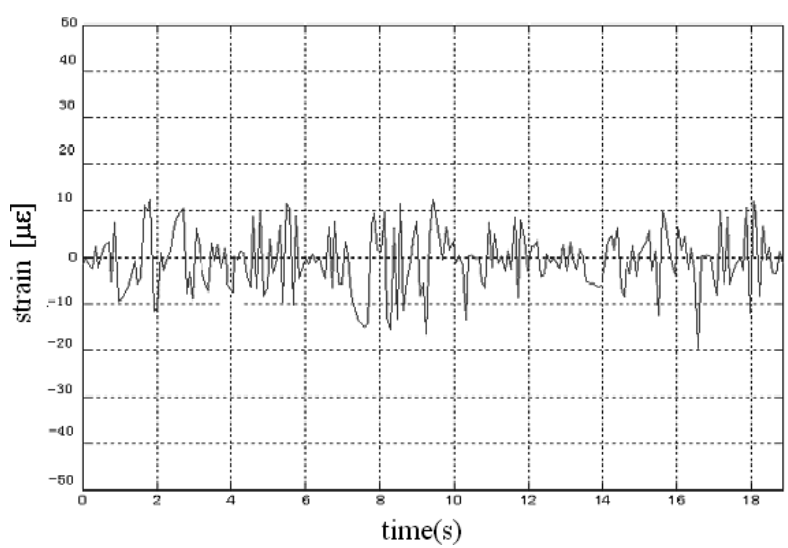

Fig. 6 Simulation time-domain dynamic response characteristics curve of the midpoint of the rocker while $\omega_{1}=$ $300 \mathrm{rpm}$

method according to the dynamic equation presented in this paper. The computer simulation curves of the time domain responses of the midpoint of coupler and rocker while the angular velocity of link $L_{1} \omega_{1}=300 \mathrm{rpm}$ are shown in Figs. 5 and 6 respectively. The exciting force is mainly the self-excitation inertia force. Because the self-excitation inertia force of the midpoint of coupler is greater than that of midpoint of rocker, the dynamic responses of the coupler midpoint are greater than that of the rocker midpoint obviously.

\section{Conclusions}

Because the 3-dimensional braided composite materials overcome the shortcomings of the two-dimensional composite laminates (such as low strength between the layers), they are the ideal materials used in high-speed linkage mechanisms. The stiffness and damping characteristics of this material linkage are analyzed according to the microstructure of the 3-dimensional braided composite materials, and the dynamic equation of the mechanism is established by the FEM. The dynamic equation includes the microstructure parameters and constituent material parameters of the composite materials and structural parameters of the linkage. The work presented in the paper provides theoretical basis to a certain extent for the further research on nonlinear vibration characteristics and optimum design of this kind of mechanism.

\section{Acknowledgment}

This research is supported by the National Natural Science Foundation of China under Grant Number 50175031 and 50565001, the Key Project of Chinese Ministry of Education under Grant Number 205119, the New Century Ten, Hundred and Thousand Talent Project Special Foundation of Guangxi under Grant Number 2003203 and the Guangxi Science Foundation under Grant Number 0542005. The authors gratefully acknowledge these support agencies. 


\section{References}

(1) Thompson, B.S., An Experimental and Analytical Study of the Dynamic Response of a Linkage Fabricated from a Unidirectional Fiber-Reinforced Composite Laminate, ASME Journal of Mechanisms, Transmissions, and Automation in Design, Vol.105, No.3 (1983), pp.526-533.

( 2 ) Thompson, B.S. and Sung, C.K., A Variational Formulation for the Dynamic Viscoelastic Finite Element Analysis of Robotics Manipulators Constructed from Composite Materials, ASME Journal of Mechanisms, Transmissions and Automation in Design, Vol.106, No.2 (1984), pp.183-190.

( 3 ) Sung, C.K. and Thompson, B.S., Material Selection: An Important Parameter in the Design of High-Speed Linkage, Mechanism and Machine Theory, Vol.19, No.4 (1984), pp.389-396.

( 4 ) Oliver, J.H., The Synthesis of Flexible Linkage by Balancing the Tracer Point Quasi-Static Deflections Using Microprocessor and Advanced Materials Technologies, Mechanism and Machine Theory, Vol.20, No.2 (1985), pp.103-104.

( 5 ) Liao, D.X. and Thompson, B.S., The Design of Flexible Robotics Manipulators with Optimal Materials Properties, The International Journal of Robotics Research, Vol.6, No.3 (1987), pp.116-129.

( 6 ) Brand, J., Drechsler, K. and Richter, H., The Application of 2-D and 3-D Woven Thermoplastic Fiber Performs for Aerospace Components, Edited by Stinson, J., 36th International SAMPE Symposium, California, April 15-18, (1991), pp.92-98.

( 7 ) Timothy, D. and Chou, T.W., Microstructural Design of Advanced Multi-Step Three-Dimensional Braided Performs, Journal of Composite Materials, Vol.28, No.13 (1994), pp.1180-1200.

( 8 ) Gong, J.C. and Sankar, B.V., Impact Properties of Three-Dimensional Braided Graphite/Epoxy Composites, Journal of Composite Materials, Vol.25, No.6 (1991), pp.715-729.
( 9 ) Yang, J.M., Ma, C.L. and Chou, T.W., Fiber Inclination Model of Three-Dimensional Textile Structural Composites, Journal of Composites, Vol.20, No.9 (1986), pp.472-483.

(10) Cox, B.N., Delamination and Buckling in 3D Composites, Journal of Composite Materials, Vol.28, No.12 (1994), pp.1114-1126.

(11) Ganwei, C., Analyses of the Stiffness and Damping of the 4-Step Three-Dimensional Braided Composite Links, Journal of Mechanical Strength, Vol.21, No.1 (1999), pp.19-23.

(12) Ganwei, C., Modal Damping of Mechanisms Fabricated from Three-Dimensional Braided Composite, China Mechanical Engineering, Vol.11, No.5 (2000), pp.481-501.

(13) Chou, T.W., Microstructural Design of Fiber Composite, (1992), Cambridge University Press, New York.

(14) Nath, P.K. and Ghosh, A., Kineto-Elastodynamic Analysis of Mechanisms by Finite Element Method, Mechanism and Machine Theory, Vol.15 (1980), pp.179197.

(15) Nath, P.K. and Ghosh, A., Steady State Response of Mechanisms with Elastic Links by Finite Element Method, Mechanism and Machine Theory, Vol.15 (1980), pp.199-211.

(16) Meriovitch, L., Element of Vibration Analysis, (1975), McGraw-Hill, Inc., New York.

(17) Daniel, I.M. and Ishai, O., Engineering Mechanics of Composite Materials, (2006), Oxford University Press, Inc., New York.

(18) Ni, R.G. and Adams, R.D., The Damping and Dynamic Module of Symmetric Laminated Composite BeamsTheoretical and Experimental Results, J. Composite Materials, Vol.18, No.2 (1984), pp.104-121.

(19) Lin, D.X., Ni, R.G. and Adams, R.D., Prediction and Measurement of the Vibrational Damping Parameters of Carbon and Glass Fiber Reinforced Plastic Plates, J. Composite Materials, Vol.18, No.2 (1984), pp.132152. 